\title{
Image Compression using Combined Approach of EZW and LZW
}

\author{
M.Vasavi ${ }^{1}$, CH.Gayatri ${ }^{2}$,M.Ravindra ${ }^{3}$, J.Ramesh reddy ${ }^{4}$, K.Santosh ${ }^{5}$ \\ ${ }^{2}$ Assistant Professor, Department of Electronics and Communication Engineering, Lendi Engineering College, \\ Vizianagaram, Andhra Pradesh- 535002, India
}

\begin{abstract}
:
Image Processing is most popular and widely used technique. In this paper we had proposed a technique for image compression. Here the user can return the processed image to the original image without any loss. In this proposed technique the test images are processed through the image compression algorithm. Here we apply combined approach of EZW and LZW. This technique is implemented on different types of images like .bmp, .tiff,.dcm (medical images), binary images. Performance of the proposed technique can be evaluated compared to LBG techniques by the parameters like PSNR, Compression ratio and MSE.
\end{abstract}

Keywords: EZW, LZW,LBG, DWT, PSNR, Compression Ratio, Binary Images, DCM Images.

\section{INTRODUCTION}

Image compression is a technique which is used to encode an image in fewer bits than it actually takes or requires. The main inflection of image compression is to decrease the redundancy of the image and to save or send data through a network in an efficient manner. In some encoding techniques, knowledge of the application is necessary to select information to discard, thereby lowering its bandwidth and intelligence. When the resulting compressed data is decoded, the result probably will not be identical to the original input, but is almost similar for that particular application. Representing the compressed data in Frequency domain is important, since most of the signal processing is done at frequency domain. One of the technique is to use DWT Discrete Wavelet Transform, where a wavelet, which is an oscillation of amplitude of the signal which starts at zero, increases and decreases again to zero. A Discrete wavelet is one where the wavelets are sampled and quantized.

\subsection{Need of compression:}

A de-compressed picture involves expansive measure of memory away media, and it sets aside greater opportunity to exchange starting with one device then onto the next. So in the event that we need to exchange or store advanced picture then we need to compress it first for quick speed of exchange and to store in a less space. Subsequently compression is exceptionally basic for present day application. A portion of the wavelets are Haar wavelet, where sequence of square functions together shaping a wavelet family and it is like Fourier examination which is spoken to as far as an orthonormal function; Bi-orthogonal wavelet, where related wavelet change is invertible but not really orthogonal and encourages more opportunity than orthogonal wavelets. It provides the possibility to build symmetric wavelet functions; Daubechies wavelets are a group of orthogonal wavelets which characterizes a DWT and portrayed by a maximal number of vanishing minutes.

\section{EZW ALGORITHM}

The main advantage of EZW encoding is that it visually improves the compressed image by increasing the decomposition level by 8 . The EZW encoder was originally designed to operate on images but it can also be used on other signals such as audio signal. It is based on progressive encoding to compress an image into a bit stream with better accuracy. It means, when more bits are added to the stream, the decoded image will contain more details. The four key ideas in the EZW algorithm are

1) Discrete wavelets transform.

2) Prediction of the absence of significant information across scales by exploiting the selfsimilarity inherence in images.

3) Entropy-coded progressive estimation quantization.

4) Universal lossless data compression accomplished by means of adaptive arithmetic coding.

A wavelet transform transforms any signal into wavelet coefficients. Each coefficient is assigned a significance symbols $(\mathrm{P}, \mathrm{N}, \mathrm{Z}, \mathrm{T})$, by comparing with the actual threshold. If the absolute value of the coefficient is greater than threshold $\mathrm{T}$ and is positive, then the coefficient is assigned as $\mathrm{P}$ and if the absolute value of the coefficient is greater 
than threshold $\mathrm{T}$ and is negative, then those coefficient is assigned as $\mathrm{N}$. If the absolute value of the coefficient is lower than threshold $\mathrm{T}$ and has only insignificant descendants, then the coefficient is assigned as $\mathrm{T}$. If the absolute value of the coefficient is lower than threshold $\mathrm{T}$ and has one or more significant descendants, then the coefficient is assigned as $\mathrm{Z}$. Then those co-efficients which are present are then there to be quantized using an entropy-coded successive approximation technique and the quantized data is compressed using a universal lossless data compression using an adaptive arithmetic coding.

\section{ALGORITHM:}

The EZW algorithm is based on four key concepts:

1. Discrete wavelet transform(hierarchical sub band decomposition)

2. Prediction of the absence of significant information across scales by exploiting the selfsimilarity inherent in images.

3. Entropy-coded successive-approximation quantization.

4. "Universal" lossless data compression which is achieved via adaptive arithmetic coding.

\section{LBG APPROACH}

This algorithm forms the basis of most vector quantizer designs. It is popularly known as the Linde-Buzo-Gray or LBG algorithm. Linde, Buzo and Gray described a technique called splitting technique for initializing the design algorithm.

\subsection{METHODOLOGY}

1) Convert $\mathrm{RGB}$ image into $\mathrm{YCbCr}$ color format

2) Take only $\mathrm{Y}$ component of $\mathrm{YCbCr}$ image, which will be the input image.

3) Input image of size $\mathrm{N} \mathrm{X} \mathrm{N}$ is divided into sub blocks of size $\mathrm{n} \times \mathrm{n}$.

4) Define the size of codebook and randomly select $\mathrm{M}$ vectors from the input vectors.

$$
d(X, C)=\sqrt{\sum_{t=1}^{k}(X t-C t)^{2}}
$$

Search the nearest code vector from $\mathrm{C}$ and then add the input vector into corresponding cluster of the closest code vector found.

5) For each code vector in the current codebook $C$, find the centroid of its associated cluster and take the centroid as a new code vector for the next iteration. Repeat step 2 and 3 till code vector don't change or change is small.

6) Measure the performance parameters PSNR and MSE of the reconstructed RGB image.

The above algorithm is executed with the different codebook size of 128, 512 and 1024 for the analysis of the parameters and effect of codebook size on the reconstructed image.

\subsection{MAIN BLOCKS OF EZW:}

\section{EZW BLOCK DIAGARAM}

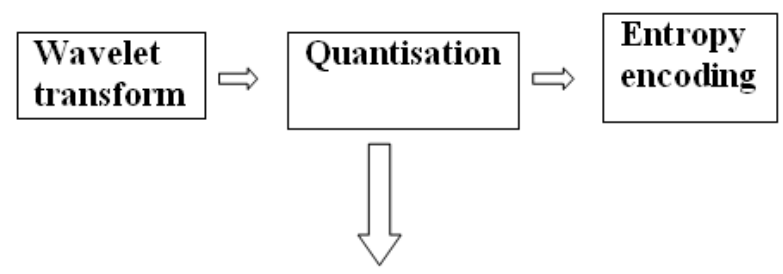

Place where embedding

takes place in ezw algorithm.

In the first block, the wavelet transform is applied on the input image and the number of stages to which the image is transformed is determined. The second block is the place where quantization takes place and hence some loss of data takes place.

This block produce certain number of levels, where decoding can be stopped at any stage depending on the thresholds for bit rate. However, the output of this block is a set of symbols that are entropy encoded by block three. The final bit stream has the property of progressive bit stream.

\subsection{About LZW:}

A different approach to adaptation is taken by the popular Lempel-Ziv-Welch (LZW) algorithm. This method was developed originally by Ziv and Lempel, and subsequently improved by Welch.

The algorithm is designed so that the string table or it may be an image can be reconstructed by the decoder based on information in the encoded stream.

The encoder executes the following steps:

1. It transmits the N-bit code for the sequence $\mathrm{S}$. 
2. It adds a new entry to the string table for $S+b$. If the encoder finds the table full when it goes to add an entry, it reinitializes the table before the addition is made.

3. It resets $S$ to contain only the byte $b$.

Where ' $S$ ' is a sequence and ' $b$ ' indicates the number of bytes. Here ' + ' denotes appending $b$ to S.

\section{PROPOSED WORK}

This section deals with the proposed techniques namely combined approach of (i) EZW + LZW. In this technique we make use of Huffman encoder and also the LZW Algorithm for the further compression. The results that which are obtained are placed in the section[6]. EZW is one of the wavelet based technique that which is proposed for the image compression. In this we are able to know about the "Discrete Wavelet Transform" and also about the "Inverse Discrete Wavelet Transform". The DWT command in the matlab performs a single-level one-dimensional wavelet decomposition with respect to either a particular wavelet ('wname', see wfilters for more information) or particular wavelet decomposition filters (Lo_D and Hi_D) that you specify.

Where Lo_D represents the low pass filter decomposition Hi_D represents the high pass filter decomposition Wname indicates the wavelet name Wfilter indicates the wavelet filters that we are using. The IDWT command in matlab performs a single-level one-dimensional wavelet reconstruction with respect to either a particular wavelet ('wname', see wfilters for more information) or particular wavelet reconstruction filters (Lo_R and Hi_R) that you specify.

Where Lo_R represents the low pass filter reconstruction

Hi_R represents the high pass filter re-construction
Figure 2 shows the combined approach of EZW algorithm, which uses a Wavelet Transform. Here, EZW algorithm is applied on the input image, and then if the output image is RGB in nature, it is then given to the LZW algorithm for the image compression and then we get the processed image after EZW and LZW decoding.

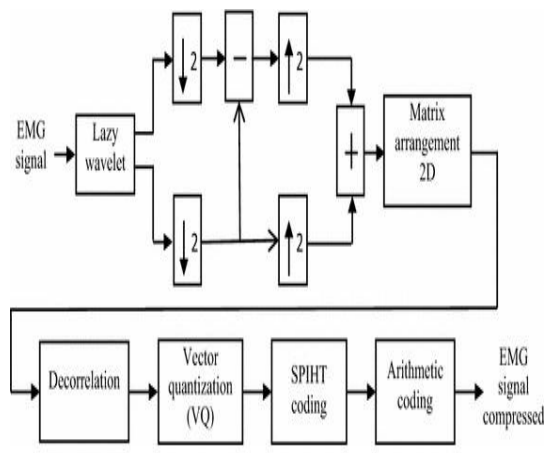

Figure 2: EZW + LZW

For further encoding that means for the further processing we process the ezw encoded signal to the lzw encoder and then we are able to decode the image with in a less time.

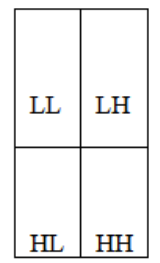

\begin{tabular}{|l|l|l|l|}
\hline L1L1 & L1L2 & L1L3 & L1L4 \\
\hline L2L1 & L2L2 & L2L3 & L2L4 \\
\hline L3L1 & L3L2 & L3L3 & L3L4 \\
\hline L4L1 & L4L2 & L4L3 & L4L4 \\
\hline
\end{tabular}

Fig 3: Image sub-bands after single-level image decomposition for (a) scalar wavelets (b) multi wavelets.

\begin{tabular}{|c|c|c|c|c|c|c|c|}
\hline 64 & -34 & 49 & 10 & 7 & 13 & 11 & 3 \\
\hline-31 & 23 & 14 & -13 & -5 & 0 & 7 & 9 \\
\hline 15 & 14 & 3 & -12 & 10 & -3 & 5 & -7 \\
\hline-9 & -7 & -14 & 8 & 4 & -2 & 3 & 2 \\
\hline-5 & 9 & -1 & 2 & 4 & 6 & -2 & 3 \\
\hline 3 & 0 & -3 & 2 & 5 & -9 & 10 & 1 \\
\hline 2 & -3 & 6 & 4 & 3 & 4 & -8 & 2 \\
\hline 5 & 11 & -6 & 5 & 9 & -2 & 1 & 0 \\
\hline
\end{tabular}

The above table shows the example for the

compression technique. Here we need $\mathrm{t}$ apply some 
threshold value to the process. The algorithm makes $2^{\wedge} \mathrm{N}$ iterations in-order to reduce the redundancy. It quantizes the bits and then reduces the usage of the common bits in the image while encoding takes place. If we consider the threshold to be 5 then $2^{\wedge} 5$ iterations are done in-order to reduce the redundancy. As if we consider the above table with the fig- 3 we are able to consider the multi wavelet block.

Here the threshold value is got considered and compared with the values present in the first block. If there is any pixel that which exceeds the threshold then that pixel is got considered as a valued pixel and it is then stored. The process will be continued until the pixels in the first block are computed, the pixels with higher value are considered. For the second iteration the block-2 is considered and now the threshold value got decreased by 1 that means the value becomes 4 . So we are able to make $2^{\wedge} 4$ iterations. Now at first the pixels that which are remained in the first block are considered and the threshold value is compared with those values then after that tha block-2 is considered and iterations starts. The process continues until we get the required values.

\section{RESULTS AND DISCUSSION}

Results of existing and proposed system are obtained using MATLAB. Here we take the 'test9.bmp' image as the input for the algorithm and is shown in figure 5.The output obtained for the Discrete Wavelet Transform compression technique using combined approach of EZW and LZW Algorithm is shown in Figure 4.

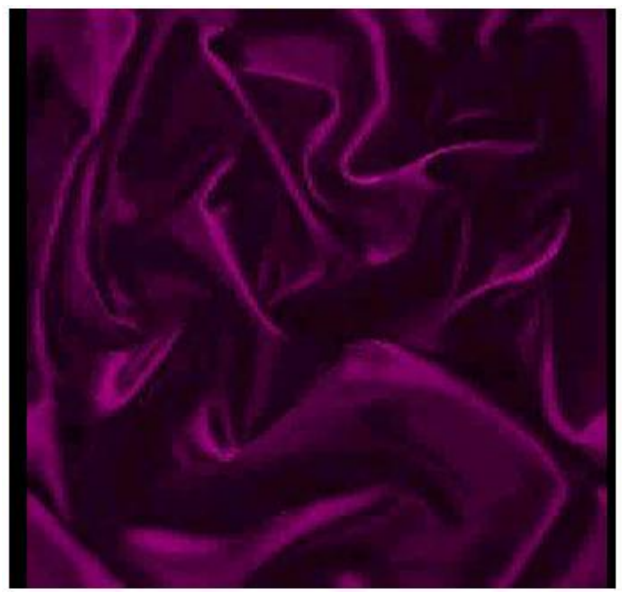

Figure 4: Processed Image

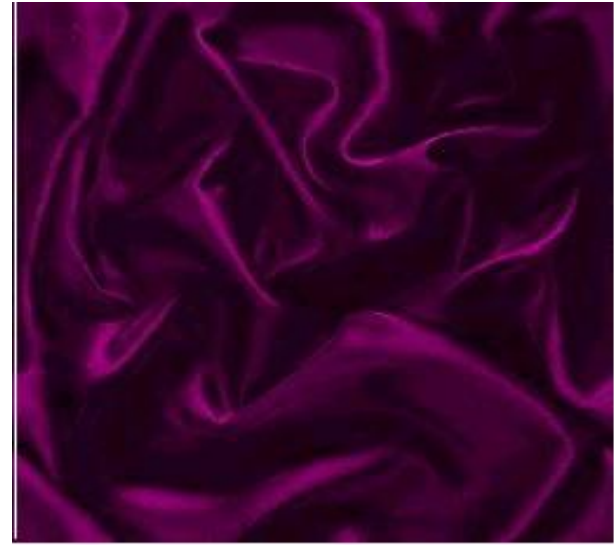

Figure 5: Original image

The above figure shows the original image and the processed test image. To what extent the image has compressed can be known with the help of compression ratio. Peak signal to noise ratio and the Mean square error is also taken with the help of below formula's

FORMULA-1:

In order to calculate the MEAN SQUARE ERROR

error = original image- (processed image);

MSE $=\operatorname{sum}\left(\operatorname{sum}\left(\right.\right.$ error $*^{*}$ error $\left.)\right) /(M *$ N)

In the above formula where $\mathrm{M}, \mathrm{N}$ represents the size of the image. Or it may be calculated mathematically by using the below formula

$$
M S E=\frac{1}{M N} \sum_{y=1}^{M} \sum_{x=1}^{N}\left[I(x, y)-I^{\prime}(x, y)\right]^{2}
$$

where $I(x, y)$ is the original image, $I^{\prime}(x, y)$ is the approximated version (which is actually the decompressed image) and $\mathrm{M}, \mathrm{N}$ are the dimensions of the images. A lower value for MSE means lesser error.

\section{FORMULA-2:}

In order to calculate the Peak signal to noise ratio $\mathrm{PSNR}=20 * \log 10(\max (\max (\max ($ origImg $))))$ $10 * \log 10(\mathrm{MSE})$.

It can also be calculated mathematically as $\mathrm{PSNR}=20 * \log 10(255 / \operatorname{sqrt}(\mathrm{MSE}))$

The inverse relation between the MSE and PSNR, this translates to a high value of PSNR means low loss.

FORMULA-3: 
To calculate the compression ratio

$\mathrm{CR}=$ Original image/processed image size.

\begin{tabular}{|l|l|}
\hline MSE & 28.18 \\
\hline PSNR & 30.89 \\
\hline Compression ratio & 7.13 \\
\hline
\end{tabular}

Table-1:The above table shows the performance characteristics of the test image using the proposed technique.

The proposed technique not only compress the gray scale images but it also used to compress the colour images. Here we are able to check the results in accordance to the ratio that which the input that is the original image is compressed.

\subsection{LBG APPROACH:}

By applying the LBG approach to the original image it compress the original image and the processed image that is the image that is decompressed is as shown the below figures .

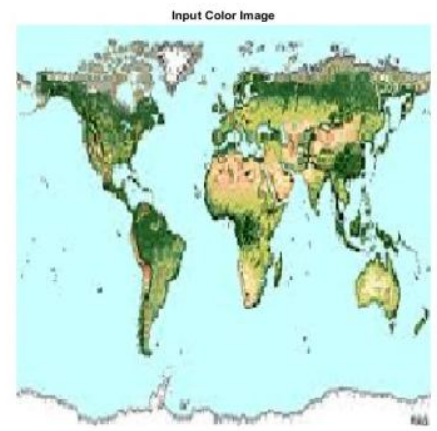

Fig-6Original test image

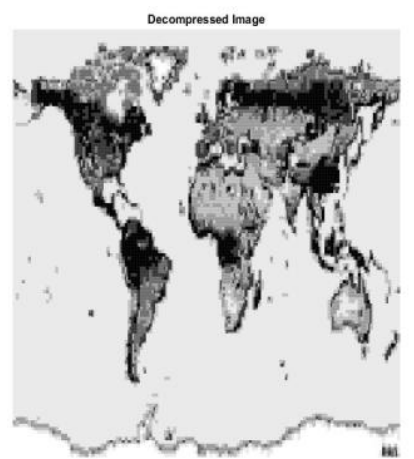

Fig-7: Decompressed image

By comparing the original image and the decompressed image we can check that the clarity of the image is not good. It means that the processed image is not as same as the original image. This technique didnot recover the original image.

\begin{tabular}{|l|l|}
\hline MSE & 220.3 \\
\hline PSNR & 19.8 \\
\hline Compression ratio & 1.6 \\
\hline
\end{tabular}

Table-2: The table shows the performance characteristics of the test image using lbg technique.

5.3 Comparison of the map test image with LBG approach and the proposed technique. Here we again consider the test map image that which acts as a input for the lbg approach.

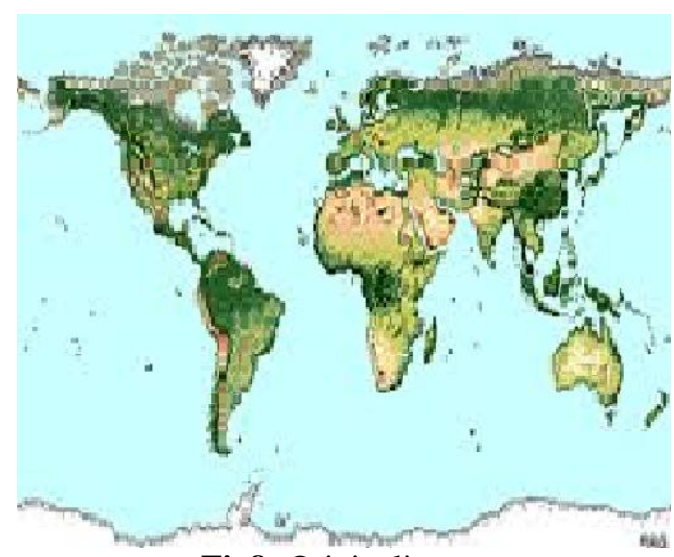

Fig8: Originalimage

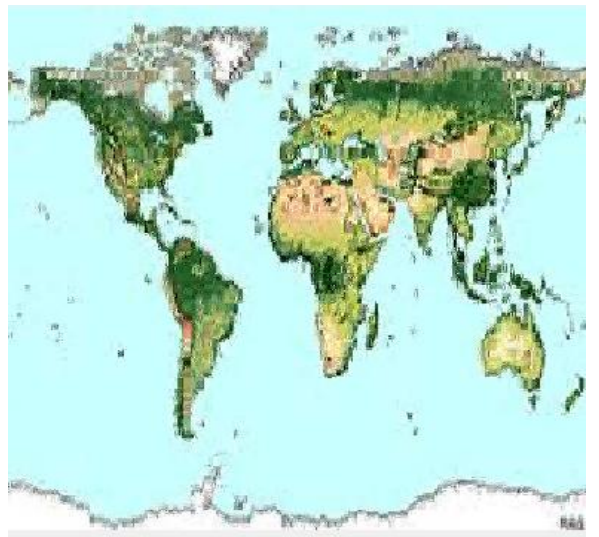

Fig-9: Processed image

\begin{tabular}{|l|l|}
\hline MSE & 283.81 \\
\hline PSNR & 23.60 \\
\hline Compression ratio & 2.31 \\
\hline
\end{tabular}

Table-3: Performance evaluation for the map image using the proposed technique.

\subsection{DCM IMAGE COMPRESSION.}

The proposed technique(EZW+LZW) is also used to compress the DILATED CARDIOMYOPATHY images (.dcm images). 


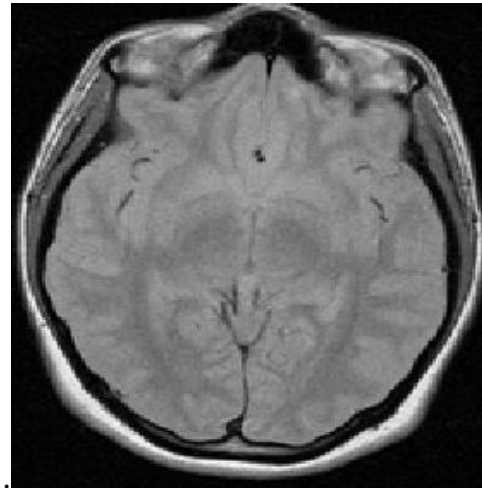

Fig: 7 Original .dcm image

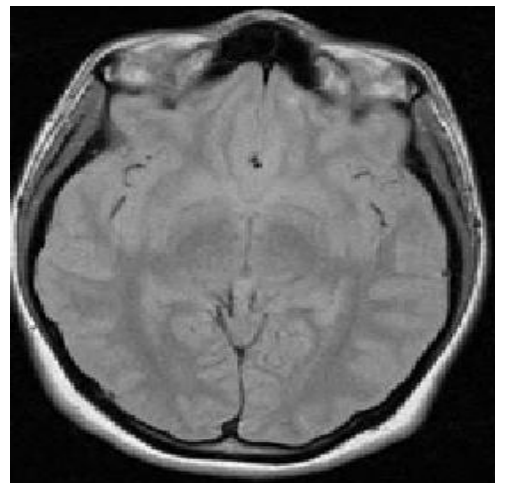

Fig:8 Processed .dcm image

\begin{tabular}{|l|l|}
\hline MSE & 59.00 \\
\hline PSNR & 41.33 \\
\hline Compression ratio & 19.88 \\
\hline
\end{tabular}

Table-4: The above results shows that the proposed technique can compress the. $\mathrm{dcm}$ images to high context and can also retrieve the original image with small losses.

\section{CONCULSION}

With this paper we came to conclude that the proposed technique is able to compress the image with in a short span of time compared to other TREE Algorithms. We are able to have compression ratio's for different images like for gray scale images, we have obtain compression ratio as $3 \%$ to $12 \%$ and for colour images including maps $2 \%$ to $7.1 \%$ and for .dcm images $19.8 \%$ to $35.6 \%$.

\section{REFERENCE}

[1]. Ms.Asmita A.Bardekar, Mr. P.A.Tijare "Implementation of LBG Algorithm for Image Compression" International Journal of Computer Trends and Technology- volume2Issue2- 2011.

[2]. Pallavi N. Save "An Improved Image Compression Method using Vector Quantization for Color Images"
[3]. International Journal of Computer Applications (0975 - 8887) ,International Conference on Computer Technology (ICCT 2015).

[4]. Aslam Khan Sanjay Mishra " Image Compression using Growing Self Organizing Map Algorithm” IJCSNS International Journal of Computer Science and Network Security, VOL.14 No.11, November 2014.

[5]. Ms. Asmita A.Bardekar\#1, Mr. P.A.Tijare\#2 "A Review on LBG Algorithm for Image Compression" (IJCSIT) International Journal of Computer Science and Information Technologies, Vol. 2 (6), 2011, 2584-2589.

[6]. R. Sivarajan, B. Elango, P. Vedasundaravinayagam "Image Compression using Combined Approach of EZW and SPIHT with DCT" International Journal of Science and Research (IJSR) ISSN (Online): 2319-7064.

[7]. Janaki. R Dr.Tamilarasi.A "Visually Improved Image Compression by using Embedded Zero-tree Wavelet Coding" IJCSI International Journal of Computer Science Issues, Vol. 8, Issue 2, March 2011.

[8]. Embedded Zerotree Wavelet - An Image Coding Algorithm Shufang Wu http://www.sfu.ca/ vswu.

[9]. "EMBEDDED IMAGE CODING USING ZEROBLOCKS OF SUBBAND/WAVELET COEFFICIENTS AND CONTEXT MODELING" Shih-Ta Hsiang and John W. Woods Center for Image Processing Research.

[10]. Akhilendra Yadav, M. A. Ansari, Manoj Tripathy," Improvement in Coding Time of Embedded Zero Wavelet Tree" International Journal of Computer Applications (0975 8887) Volume 49- No.3.

[11]. Rehna V. J and Jeya Kumar M. "WAVELET BASED IMAGE CODING SCHEMES: A RECENT SURVEY". International Journal on Soft Computing (IJSC) Vol.3, No.3

[12]. Arber Borici, Saif Alzahir, "An Innovative Lossless Compression Method for Discrete-Color Images", IEEE TRANSACTIONS ON IMAGE PROCESSING. 